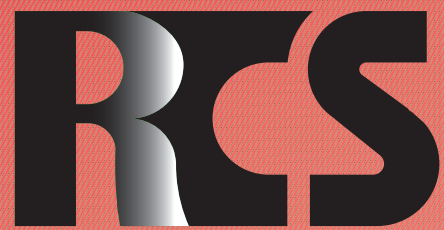

Depósito legal ppi $201502 Z U 4662$

Esta publicación científica en formato digital es continuidad de la revista impresa Depósito Legal: pp $197402 Z$ Z789

- ISSN: 1315-9518 • ISSN-E: 2477-9431

Revista de Ciencias Sociales

Universidad del Zulia. Revista de la Facultad de Ciencias Económicas y Sociales

Vol. XXVII,

No. Especial 3, 2021

Esta publicación científica en formato digital es continuidad de la revista impresa Depósito Legal: pp $197402 Z$ Z789

ISSN: $1315-9518$ 


\title{
Gestión integrada de recursos hídricos y gobernanza: Subcuenca del río Vinces, provincia Los Ríos-Ecuador
}

\author{
Muñoz Marcillo, José Luis* \\ Bustos Cara, Roberto**
}

\section{Resumen}

En el presente trabajo se abordó la gestión integrada de recursos hídricos-territoriales y gobernanza de la cuenca del río Vinces, que forma parte de la gran cuenca del río Guayas aplicado a los cambios del uso del suelo y los del agua para riego agrícola. En un estudio descriptivo, se estudiaron las características físico-naturales y topográficas de la cuenca del río Vinces, centrándose en la evolución del manejo histórico del río Guayas, mapa de actores públicos y privados, así como normativa vigente para gestión integrada del manejo de cuencas hidrográficas. Se analizaron proyectos de riego existentes y usos del suelo agrícola de la zona alta, media y baja del río Vinces complementados con análisis de demanda de agua para riego agrícola. Asimismo, se analizaron las limitaciones del sistema de administración estatal, demostrando que existe una alta demanda de agua para riego agrícola de monocultivos, cuya mayor tasa de productividad coincide con el verano del país, por lo que la presión sobre los cursos hídricos superficiales los coloca en déficit critico. Se concluye, que existe falencias en la actual gestión de las cuencas hidrográficas del Ecuador, siendo necesario aplicar modelos de gobernanza integrados para un manejo sostenible de las mismas.

Palabras clave: Recursos hídricos; gobernanza; cuenca hidrográfica; río Guayas; río Vinces.

\footnotetext{
* Magíster en Geografía mención Recursos Territoriales. Ingeniero Forestal. Docente de la Universidad Técnica Estatal de Quevedo, Ecuador. E-mail: jsmunoz@uteq.edu.ec iD ORCID: https://orcid. org/0000-0001-9744-3745

** Doctor en Geografía. Docente de la Universidad Nacional del Sur, Argentina. E-mail: usbustos@uns. edu.ar ORCID: iD https://orcid.org/0000-0001-9205-8792
} 


\title{
Integrated management of water resources and governance: Vinces river sub-basin, Los Ríos Province-Ecuador
}

\begin{abstract}
In the present work, the integrated management of water-territorial resources and governance of the Vinces river basin, which is part of the great Guayas river basin, applied to changes in the use of land and water for agricultural irrigation was addressed. In a descriptive study, the physical-natural and topographic characteristics of the Vinces river basin were studied, focusing on the evolution of the historical management of the Guayas river, a map of public and private actors, as well as current regulations for integrated management of basin management. hydrographic. Existing irrigation projects and agricultural land uses in the upper, middle and lower Vinces river areas were analyzed, complemented by analysis of water demand for agricultural irrigation. Likewise, the limitations of the state administration system were analyzed, showing that there is a high demand for water for agricultural irrigation of monocultures, whose higher productivity rate coincides with the country's summer, so that the pressure on surface water courses places them in critical deficit. It is concluded that there are shortcomings in the current management of the hydrographic basins of Ecuador, making it necessary to apply integrated governance models for their sustainable management.
\end{abstract}

Keywords: Water resources; governance; watershed; Guayas River; Vinces river.

\section{Introducción}

Las problemáticas complejas de degradación de un recurso fundamental para el desarrollo del territorio, impulsan a proponer un análisis integrado de la situación del manejo de la cuenca del río Vinces, para cuyo efecto se tomó en cuenta la relación de escalas de comprensión y acción, con sus dificultades de integración. De esta manera, se aborda la escala de la cuenca del río Guayas por su extensión e importancia en términos productivos y demográficos para Ecuador. Finalmente, a escala local, la investigación se focalizó en torno a las localidades de los cantones Valencia, Quevedo y Mocache (situadas en la subcuenca del río Vinces), cuya ubicación estratégica permite individualizar diferentes realidades territoriales que describen la parte alta, media y baja de la cuenca del río Vinces, respectivamente.

Cabe destacar, que la cuenca del río Guayas, es una de las unidades territoriales más importantes del Ecuador desde el punto de vista socioeconómico, dado que ella acoge el $30 \%$ de la población del Ecuador y alberga la mayor y más diversa producción agropecuaria del país. Los problemas derivados del manejo de la cuenca del Guayas son evidentes, en especial por la sobrecarga del suelo con monocultivos intensivos que requieren riego permanente y aplicación constante de productos químicos con la consecuente degradación de los recursos hídricos.

La presente investigación analiza los antecedentes históricos en el manejo de la cuenca del río Guayas relacionados con la Gestión Integrada de Recursos Hídricos (GIRH), el manejo de los recursos territoriales, en complemento con la noción de Gobernanza, estableciendo para ese efecto una línea de tiempo y espacio en el que se da cuenta de los eventos más significativos en el ámbito de manejo administrativo que han moldeado su realidad actual. En ese sentido, "la gobernanza es vista como un proceso 
continuo de negociación y/o cooperación para la resolución de problemas, en el que ocupa la vinculación un lugar de relevancia" (Sánchez y Castañón, 2019, p.62). Asimismo, luego de la caracterización físico-natural y administrativa de la cuenca del río Guayas, se aplica un análisis en detalle a una de sus más importantes subcuencas, la cuenca del río Vinces.

Por su parte, Ramos-Soto, et al. (2020) manifiestan que: "Se entiende a la gestión integral, como el conjunto de normas y prácticas en la cual se incluye la gestión de calidad, el cuidado del medio ambiente, la prevención de riesgos laborales" (p.34), de tal manera, que puedan obtenerse beneficios como un incremento en la productividad, así como un entorno apropiado para el desarrollo de la misma.

En ese sentido, la GIRH es una filosofía que ofrece un marco conceptual de guía con una meta de gestión y desarrollo sostenible del recurso hídrico, que busca que las personas traten de cambiar sus prácticas de trabajo para que vean la totalidad del contexto que rodea sus acciones y entiendan que las mismas no existen en forma independiente de las acciones de los otros. De manera adicional, busca introducir un elemento de democracia descentralizada en la gestión del agua haciendo énfasis en la participación de los interesados y la toma de decisiones en el nivel más bajo posible (Red Internacional para el Desarrollo de Capacidades en la Gestión Integrada del Recurso Hídrico [Cap-Net] y GWP, 2005).

La Gestión Integrada de los Recursos Hídricos (GIRH), constituye el paradigma actual de la gestión del agua a nivel mundial, haciéndose explícito en políticas nacionales para la gestión del agua a nivel global. Asimismo, busca orientar el desarrollo de políticas públicas en materia de recursos hídricos, a través de una conciliación entre el desarrollo económico y social, así como la protección de los ecosistemas (Martínez y Villalejo, 2018). Al respecto, Gómez y Gómez (2013), sostienen que: "La ordenación del territorio se hace operativa a través de un conjunto de instrumentos o planes de ordenación territorial los cuales configuran un sistema coherente de planificación, es decir de previsiones de futuro sobre el sistema territorial" (p.3), a todos los niveles.

$\mathrm{La}$ GIRH, es una estrategia de adaptación en sistemas hídricos expuestos a fuertes variaciones espaciales y temporales a consecuencia del cambio climático, que hacen que la oferta de agua sea cada vez más incierta mientras la demanda crece (García, Carvajal y Jiménez, 2007).

\section{Gestión Integral de los Recursos Hídricos (GIRH)}

La Gestión Integrada de los Recursos Hídricos (GIRH), constituye un marco de trabajo cada vez más usado de manera global para evaluar y tomar decisiones en relación con el agua (Cap-Net, 2008). Este marco resulta muy útil para abordar los problemas de calidad del agua, los usos opuestos del agua y la gobernanza de la misma. La GIRH, ha sido definida como un medio que promueve y garantiza la gestión, así como provisión equitativa, económicamente sólida y ecológicamente sostenible, de los recursos hídricos mediante el desarrollo y manejo coordinados del agua, la tierra, al igual que otros recursos relacionados, con el fin de maximizar el bienestar económico y social sin comprometer la sostenibilidad de los ecosistemas vitales (Asociación Mundial para el Agua [GWP], 2000).

La misión de la GIRH, ha sido conciliar los diferentes usos del agua que están en competencia a través de la participación de las partes interesadas y la descentralización de la gobernanza a la unidad hidrológica más baja posible (Smith y Clausen, 2018). El argumento de la GIRH, es que el agua debe tratarse como un bien económico con beneficios económicos, financieros y ambientales, para la sostenibilidad de la vida en la tierra (Ngene, et. al. 2021).

La creciente escasez de agua se considera cada vez más un riesgo global a medida que el uso humano de los recursos hídricos continúa 
aumentando rápidamente, dejando menos agua para mantener la integridad ecológica (Postel, 2000; Organización de las Naciones Unidas para la Alimentación y la Agricultura [FAO], 2009; Pahl-Wostl, et al., 2013; Rockström, et al., 2017). Maximizar el área de agricultura de regadío es una importante dirección estratégica para muchos países que luchan por cumplir sus objetivos de seguridad alimentaria y erradicación de la pobreza (Rosegrant, Ringler y Zhu, 2009; Özerol, Bressers y Coenen, 2012). La creciente intensificación de la producción agrícola en gran parte del mundo, ha aumentado la presión sobre el medio ambiente, la biodiversidad y otros recursos naturales, incluida el agua (Mersha, et. al., 2021).

Por su parte, Aguirre (2011) señala que la cuenca hidrográfica brinda variados e importantes servicios vinculados con los recursos hídricos como el abastecimiento de agua para la población y para las actividades productivas, así como la provisión de servicios indirectos como los de regulación de hábitat, entre otros. Las cuencas hidrográficas, se constituyen en la unidad territorial más adecuada para el manejo integrado de los recursos hidrográficos (Dourojeanni, Jouravlev y Chávez, 2002), ubicándose como un área donde se interrelacionan los recursos naturales y el sistema socioeconómico, dotando al territorio de servicios ambientales indispensables para su desarrollo (Oficina de Evaluación y Supervisión [Ove], 2012).

"El estudio de la gobernanza históricamente ha tenido diferentes vertientes; sin embargo, hay algunas coincidencias, en las que se distingue que el Estado impone las reglas de acción” (Sánchez y Castañón, 2019, p.61). En ese sentido, la Gobernanza es definida como "la capacidad de abarcar la totalidad de las instituciones y relaciones implicadas en el proceso de gobierno, vinculando el sistema político con su entorno" (Pierre y Peters, 2000, p.1). En su expresión más general, ésta se refiere a un cambio en el equilibrio entre el Estado y la sociedad civil, en el que se coloca el acento en la ciudadanía activa y la vincula, en definitiva, a debates más amplios en torno a la democracia deliberativa, el comunitarismo y la visión neorepublicana de la sociedad civil (Natera, 2004; Contreras y Montecinos, 2019).

La gobernanza no es un esfuerzo neutral, sino cargado de poder, lo que a su vez sugiere que no se trata solo de hacer reglas sino también de lograr el poder (Lemos y Agrawal, 2006). De esta manera, la gobernanza no es solo un proceso para la toma decisiones, también es el medio para producir sujetos con las características prioritarias para la implementación efectiva de esas decisiones (Budds, 2020). De igual manera, Barthe, (2001) se refiere al concepto de gobernanza orientada a la gestión del territorio y específicamente al territorio local, manifestando que la definición de local gouvernance apareció en la sociología política y urbana anglo sajona siendo introducida en Francia con el fin de designar las nuevas modalidades de acción pública asociada, en la etapa de descentralización y la emergencia de un poder supranacional europeo.

Kooiman (2005), describe a la gobernanza como la remodelación de las actividades del gobierno y una mayor conciencia sobre la necesidad de cooperar con otros actores sociales. Es decir, a una creciente concienciación no sólo de las limitaciones del tradicional orden y control público como mecanismo de gobierno, sino también de las respuestas a los problemas sociales que requieren un mayor número de enfoques e instrumentos.

\section{Metodología}

En un estudio descriptivo, se estudiaron las características físico-naturales y topográficas de la cuenca del río Vinces, centrándose en la evolución del manejo histórico de la cuenca del río Guayas, el mapa de actores públicos y privados, así como la normativa vigente para gestión integrada del manejo de cuencas hidrográficas. Se analizaron los proyectos de riego existentes en la cuenca del río Guayas y los usos del suelo agrícola de la zona alta, media y baja de la cuenca del 
río Vinces, complementados con el análisis de la demanda de agua para riego agrícola en la cuenca del río Vinces.

En ese sentido, la cuenca del río Guayas, se extiende entre los paralelos $00^{\circ}$ $14^{\prime} \mathrm{S}, 02^{\circ} 27^{\prime} \mathrm{S}$ y los meridianos $78^{\circ} 36^{\prime} \mathrm{W}$, $80^{\circ} 36^{\prime} \mathrm{W}$, abarcando territorios parciales o totales de ocho de las veinticuatro provincias ecuatorianas: Guayas, Los Ríos, Manabí, Santo Domingo de los Tsáchilas, Cotopaxi, Bolívar, Chimborazo, y Cañar. A su vez, las provincias de Guayas y Los Ríos, representan juntas el $48 \%$ de la superficie de la cuenca y el $72 \%$ de su población. El área total cubre aproximadamente $32.219 \mathrm{~km}^{2}$ (ver Figura I).

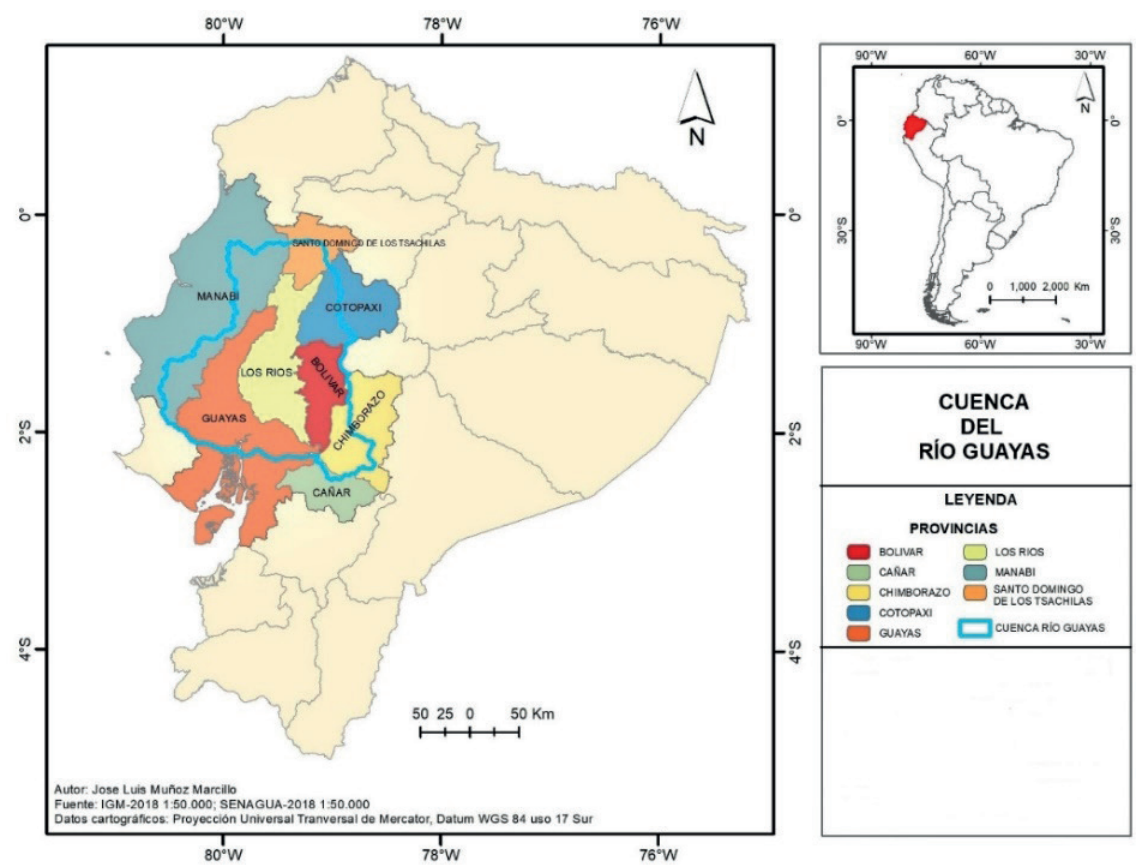

Fuente: Elaboración propia, 2021, basado en Secretaría del Agua (SENAGUA, 2017); e Instituto Geográfico Militar (IGM, 2018).

\section{Figura I: Cuenca del río Guayas}

La cuenca del río Vinces, se ubica desde el sector nor-oriental al centro de la cuenca del río Guayas, extendiéndose por 426.800 hectáreas y recorre $267,96 \mathrm{~km}$ de distancia en su eje hídrico principal, siguiendo un sentido norte-sur constituyéndose en parte importante de la cuenca del río Guayas. Abarca el 57\% de la superficie de la provincia de Los Ríos, territorio de la costa ecuatoriana eminentemente agrícola con presencia de cultivos tropicales y subtropicales de exportación como abacá, arroz, banano, café, cacao, maíz, palma aceitera, entre otros.

En ese sentido, la cuenca del río Vinces toma su nombre del río Vinces, que la atraviesa en sentido norte - sur. Río, que toma diferentes nombres a lo largo de su recorrido, de tal manera, que en la parte norte entre los 
Gestión integrada de recursos hídricos y gobernanza: Subcuenca del río Vinces, provincia Los Ríos-Ecuador

Muñoz Marcillo, José Luis y Bustos Cara, Roberto

cantones Santo Domingo de Los Tsáchilas y San Jacinto de Buena Fe, se denomina río Baba y de allí hacia el sur de la cuenca, toma el nombre del cantón por donde atraviesa, así se tiene que se denomina, río Quevedo, río
Mocache y río Vinces. El río Quevedo, se forma de la convergencia de tres ríos, siendo estos los ríos Baba, Lulo y San Pablo (ver Figura II).

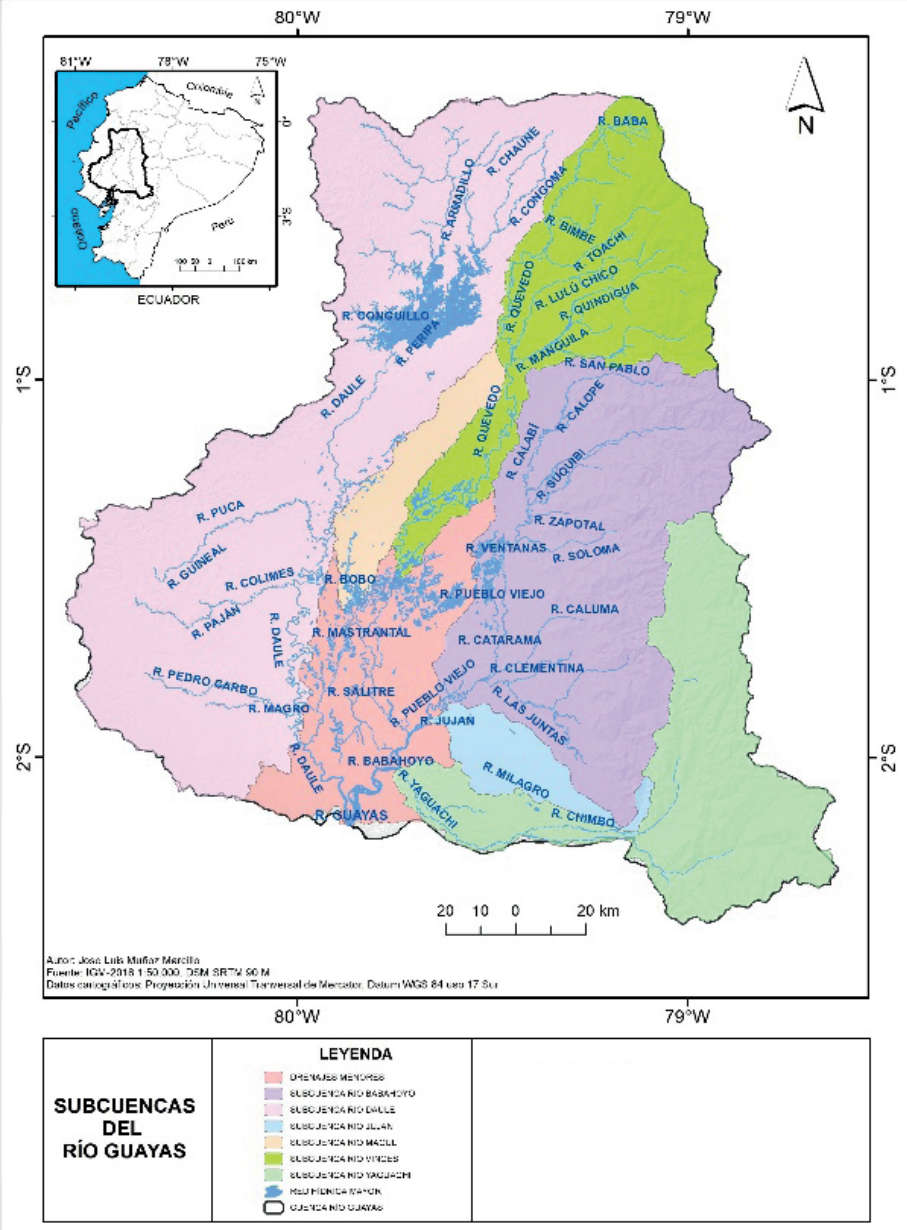

Fuente: Elaboración propia, 2021, basado en SENAGUA (2017) e IGM (2018).

Figura II: Subcuencas de la cuenca del río Guayas 
La cuenca del Guayas pertenece a la vertiente Occidental, constituye el sistema fluvial más importante de la costa sudoccidental del Pacifico, está conformada por siete subcuencas cuya red de drenaje nacen en las estribaciones occidentales de la Cordillera de los Andes y en la vertiente oriental de la Cordillera Costanera Chongón - Colonche, que conforman los ríos Daule y Babahoyo, los cuales unen sus caudales 5 kilómetros antes de la ciudad de Guayaquil dando origen al Rio Guayas, el cual tiene una longitud de $93 \mathrm{~km}$ desde La Puntilla en la provincia del Guayas hasta Punta Arenas en la Isla Puna (estuario) para desembocar al Océano Pacifico en el Golfo de Guayaquil (Instituto Oceanográfico de la Armada [INOCAR], 2010) (ver Figura II).

La cuenca del río Vinces, se ubica desde el sector nor-oriental al centro de la cuenca del río Guayas, extendiéndose por 4.268 $\mathrm{km} 2$, tomando como se dijo anteriormente, el nombre del río que la atraviesa, por lo tanto, a la altura del cantón Quevedo, se conoce como río Quevedo; luego en el cantón Mocache, se denomina río Mocache; para finalmente al atravesar los cantones Palenque y Vinces, identificarse como río Vinces (ver Figura III).

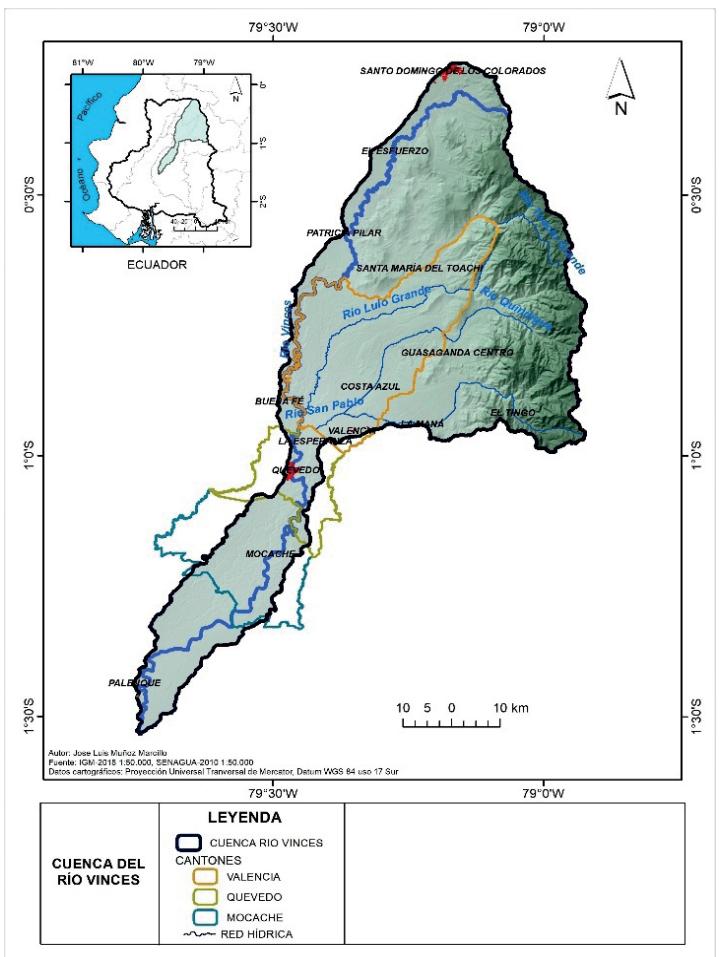

Fuente: Elaboración propia, 2021, basado en SENAGUA (2017) e IGM (2018).

Figura III: Cuenca del río Vinces 
De la misma manera, se realizó un análisis de las limitaciones que presenta el actual sistema de administración estatal de la cuenca del río Guayas y el río Vinces. Además, se realizaron mapas básicos y temáticos a escalas global, regional y local de la cuenca del río Guayas, cuenca del río Vinces, y zona alta, media y baja de la cuenca del río Vinces, respectivamente.

El Plan Nacional de Gestión Integrada e Integral de los Recursos Hídricos (Changjiang Institute of Survey Planning Design and
Research [CISPDR], 2016a; 2016b) de las Cuencas y Microcuencas Hidrográficas del Ecuador (PNGIRH), realiza un análisis para 9 Demarcaciones Hidrográficas continentales (ver Figura IV), y un análisis específico para las Islas Galápagos, con el objeto de resolver el conflicto entre oferta y demanda de los recursos hídricos, salvaguardando la vida de las personas, la soberanía alimentaria y garantizando el consumo de agua industrial. Asimismo, establece años horizonte: Consta de un año base (2010).
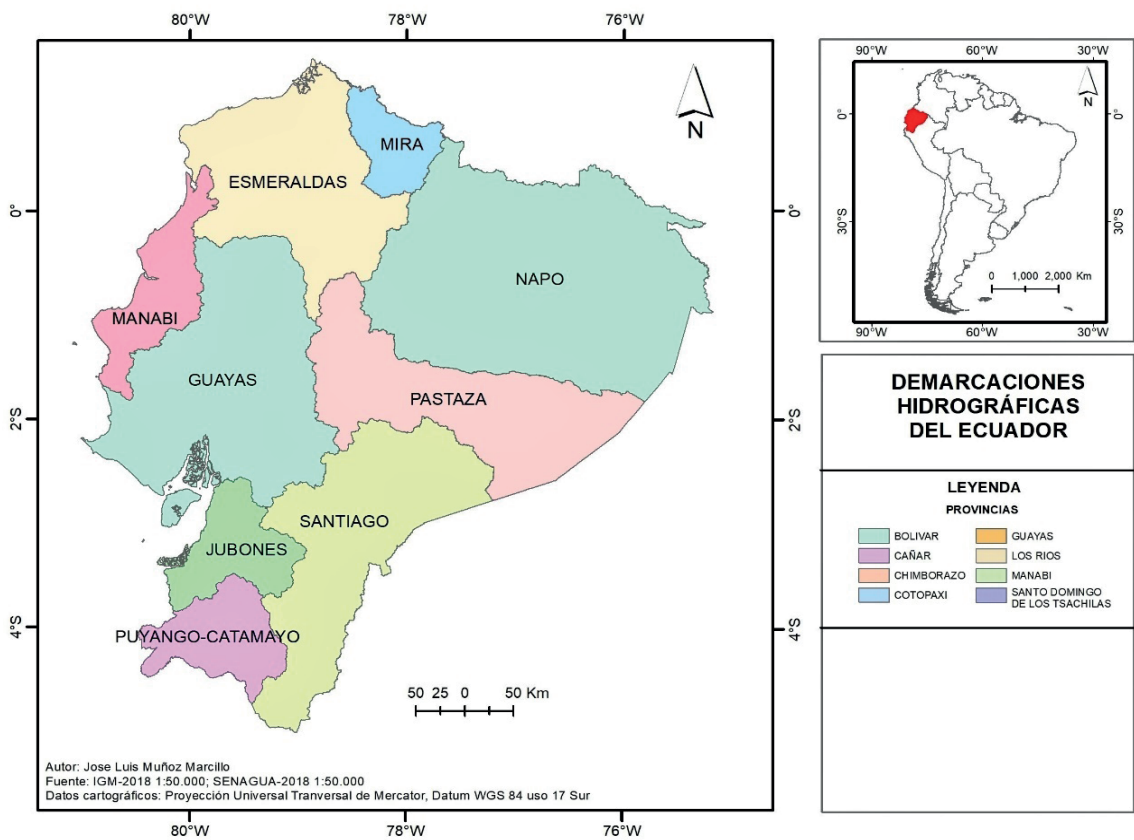

Fuente: Elaboración propia, 2021 con base en SENAGUA (2017) e IGM (2018).

\section{Figura IV: Demarcaciones Hidrográficas de Ecuador}

Para elaborar el marco conceptual se analizó la bibliografía y los documentos institucionales relacionados con la geografía y el análisis territorial, con la gestión integrada de recursos hídricos en América Latina y de manera particular en Ecuador. En ese sentido: a. Para realizar la caracterización del proceso de gestión de la cuenca hidrográfica del río Guayas en un contexto institucional, temporal, espacial y normativo, se recurrió al análisis de material bibliográfico y documentos de investigación alojados en las bibliotecas 
particulares de las instituciones ecuatorianas, así como en el repositorio de la Comisión Económica para América Latina y el Caribe (CEPAL), como el trabajo de Küffner (2004).

b. Para realizar la caracterización físicanatural y socioeconómica de la cuenca del río Guayas, se empleó información cartográfica básica y temática a escala 1:100.000, 1:50.000 y 1:25.000 provista por el Instituto Geográfico Militar (IGM), Instituto Espacial Ecuatoriano (IEE), Instituto Nacional de Estadísticas y Censos (INEC) y Ministerio de Agricultura y Ganadería (MAGAP, 2015). La información cartográfica antes mencionada se trabajó en el programa de Sistemas de Información Geográfica (SIG) ArcGIS Desktop 10.1, con el fin de estructurar e implementar la base de datos geográficos, analizar los datos y elaborar la cartografía temática.

c. Para establecer el estado actual de la gestión existente en la cuenca del río Vinces, se aplicaron entrevistas a los principales actores que guardan relación con el esquema de manejo de las cuencas en el Ecuador.

\section{Resultados y discusión}

En esta parte, se inicia con el análisis de la gran cuenca del río Guayas en donde se realiza un análisis físico de la misma, que incluye información geográfica digital básica y temática a una escala cartográfica apropiada, se exploran los problemas del uso del agua de la cuenca, los proyectos de riego, así como drenaje, que existen en la cuenca y finalmente, se caracteriza el uso y cobertura del suelo de la cuenca.

En una escala de detalle, se analiza la subcuenca del río Vinces como parte funcional de la gran cuenca del río Guayas, en donde se abordan aspectos como la caracterización física del área, los usos del agua de la misma, y se incorpora un anexo cartográfico como insumo para la GIRH. Finalmente, a escala de cantón se realiza un análisis en la parte alta, media y baja de la cuenca, del uso y cobertura del suelo predominante, contrastado con la tenencia de la tierra rural y el análisis de los usos urbanos de los ríos en los cantones cuya cabecera cantonal se encuentra influenciada por el cauce principal de la subcuenca del río Vinces.

\subsection{Gobernanza y actores políticos en el manejo de cuencas hidrográficas en Ecuador}

En el esquema de la Figura V, Bustos (2014) propone una definición simple de gobernanza como la interacción entre tres esferas de acción constructora de territorio e interviniente en la gestión del mismo. 


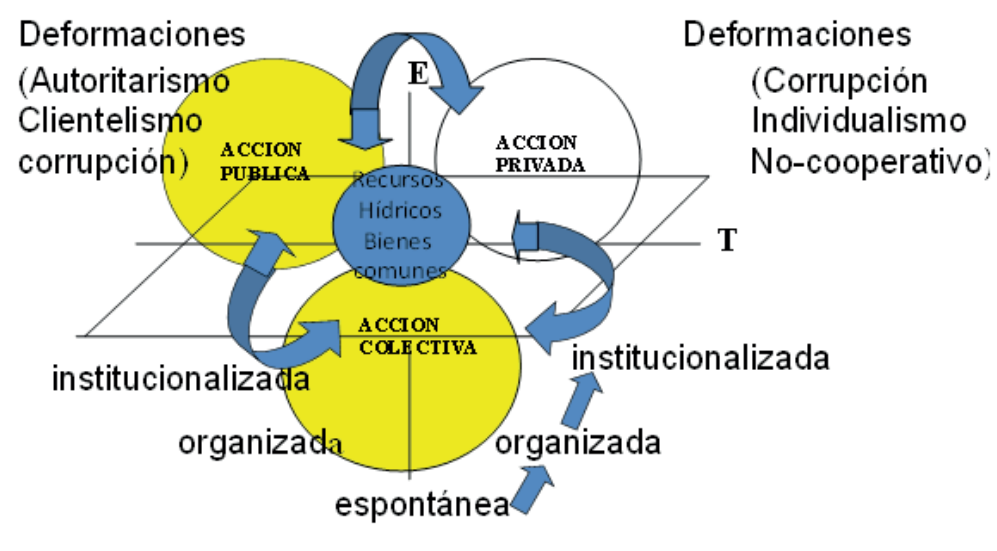

\section{Deformaciones (anomia-violencia-fragmentación)}

Fuente: Bustos (2014).

Figura V: Gobernanza

El esquema de Bustos (2014), "resume de manera simple, las tres esferas de acción de la gestión territorial, en este caso aplicado a la gestión de un recurso entendido como bien común el agua" (p.270). O mejor dicho una cuenca hidrográfica entendida como territorio. La acción pública, como acción gubernamental que, a partir de una institucionalidad específica, políticas públicas y proyectos, gobierna. La acción privada, asociada a una lógica de maximización de la ganancia y a una apropiación privada; y, la acción colectiva, con distintos grados de organización y participación en la defensa de intereses diversos. El equilibrio entre los tres componentes da una estabilidad al sistema que de todas maneras se concibe como dinámico. Este sistema de interacción en un espacio determinado, puede generar consensos, acuerdos o pactos territoriales, pero también conflictos.

La buena gobernanza del agua, aborda la asignación y gestión de los recursos hídricos $\mathrm{y}$ responde a los problemas colectivos.
Los temas críticos en la gobernanza son: Responsabilidad, participación, transparencia, efectividad, estado de derecho y equidad (Integrated Watershed Management [IWM], 2015). Por lo tanto, la gobernanza del agua se convierte en una solución sostenible cuando salvaguarda los ecosistemas, pero es capaz de considerar el bienestar social y económico de las personas (Wiek y Larson, 2012).

Existen varios factores importantes para la gobernanza sostenible del agua (Van Rijswick, et al., 2014). Estos son conocimientos sobre sistemas de agua; monitoreo y aplicación; valores, principios y discursos de políticas; responsabilidad, autoridad y medios; participación de las partes interesadas; capacidad de resolución de conflictos; compensaciones entre objetivos sociales como asignación, regulación y acuerdos; y, arreglos financieros. En la Figura VI, se presenta el mapa de actores políticos actuales en la administración de las cuencas hidrográficas en Ecuador. 


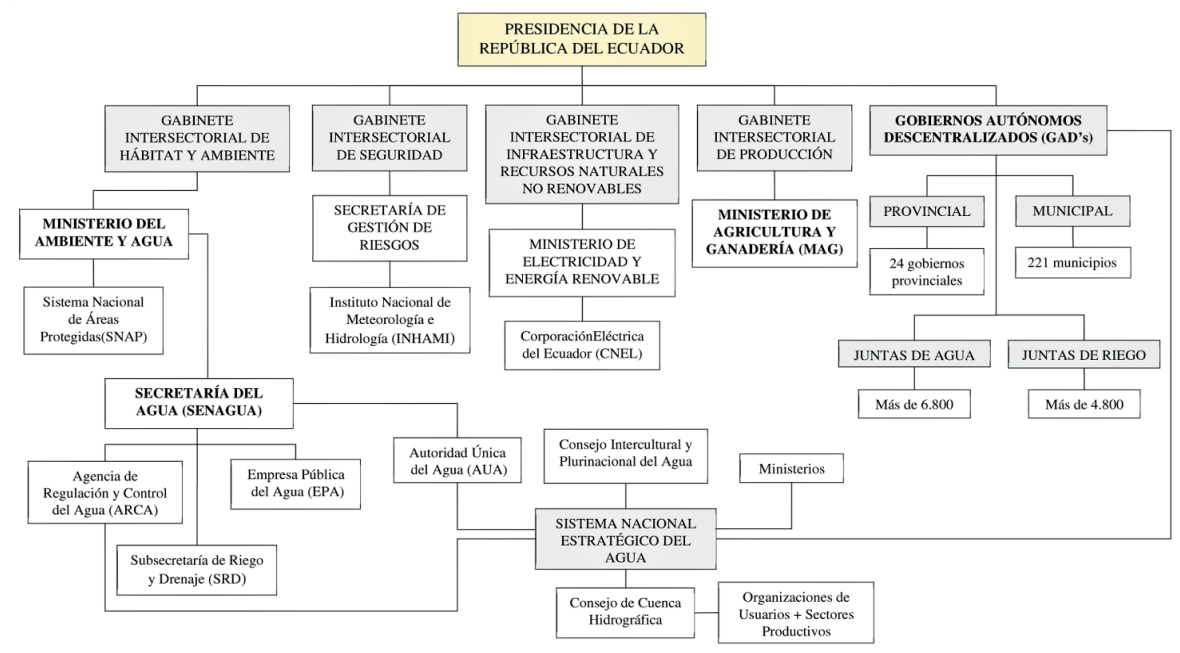

Fuente: Elaboración propia, 2021 en base a Consorcio Camaren (2012); y Secretaría Nacional de Planificación y Desarrollo (SENPLADES, 2017).

\section{Figura VI: Mapa de actores políticos actuales en la administración de las cuencas hidrográficas en el Ecuador}

\subsection{Cuenca del río Guayas}

La cuenca del río Guayas, abarca un área en que se han venido desarrollando una variedad de usos de la tierra como el agrícola, forestal y pecuario. Esto se cimenta en la importante gradiente altitudinal y en la variedad de clases de suelo existentes que caracteriza a la cuenca, lo que ha permitido el establecimiento de diversos cultivos agrícolas de ciclo corto, anuales y perennes, tanto del tipo tropical como templados (ver Figura VII y Tabla 1). El impacto del cambio de uso de la tierra incluye no solo la urbanización, sino también el desarrollo y consolidación de la agricultura (Wheater y Evans, 2009). El crecimiento de la población y el cambio climático son cargas añadidas a la crisis mundial del agua (Secretaría Nacional del Agua y Servicio Alemán de Cooperación Técnico Social [SENAGUA-DED], 2009; Kumar, et. al., 2021). 
Gestión integrada de recursos hídricos y gobernanza: Subcuenca del río Vinces, provincia Los Ríos-Ecuador

Muñoz Marcillo, José Luis y Bustos Cara, Roberto

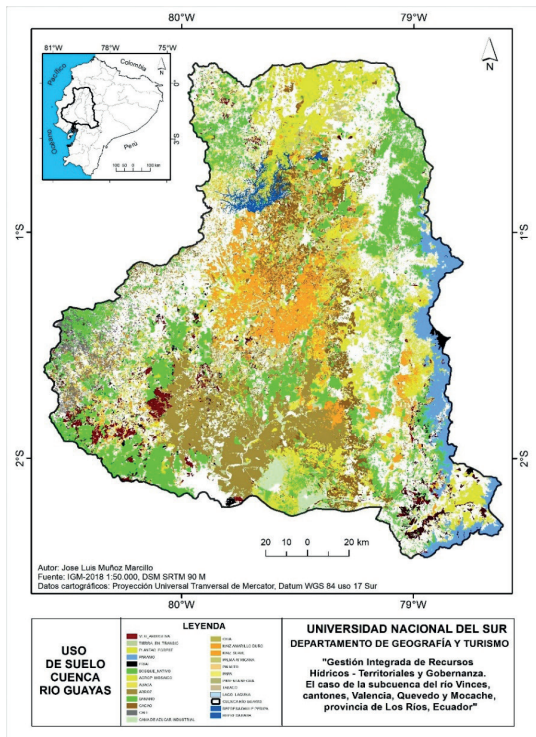

Fuente: Elaboración propia, 2021.

Figura VII: Uso del suelo en la cuenca del Guayas

Tabla 1

Uso agrícola del suelo de la cuenca del Guayas

\begin{tabular}{lcc}
\hline \multicolumn{1}{c}{ Uso Agrícola } & Área (HA) & \% \\
\hline Pimienta Negra & 14.083 & 0,001 \\
Chía & 58.134 & 0,006 \\
Abacá & $1.701,840$ & 0,176 \\
Palmito & $1.717,553$ & 0,178 \\
Tabaco & $3.238,843$ & 0,335 \\
Papa & $3.787,973$ & 0,392 \\
Caña de Azúcar Industrial & $38.115,807$ & 3,942 \\
Café & $44.983,962$ & 4,652 \\
Palma Africana & $58.535,177$ & 6,053 \\
Banano & $86.790,439$ & 8,975 \\
Cacao & $176.616,968$ & 18,265 \\
Maíz Amarillo Duro & $277.715,08$ & 28,720 \\
Arroz & $273.701,471$ & 28,305 \\
TOTAL & $\mathbf{9 6 6 . 9 7 7 , 3 3 1 4}$ & $\mathbf{1 0 0 , 0 0}$ \\
\hline
\end{tabular}

Fuente: Elaboración propia, 2021. 
Desde la década de los '60 hasta la actualidad en Ecuador, se han ido incorporando una serie de instituciones públicas para el manejo de las cuencas hídricas, varias de estas instituciones luego han sido eliminadas o absorbidas por otras entidades, de acuerdo a lo que ha decidido el gobierno de turno (ver Figura VIII).

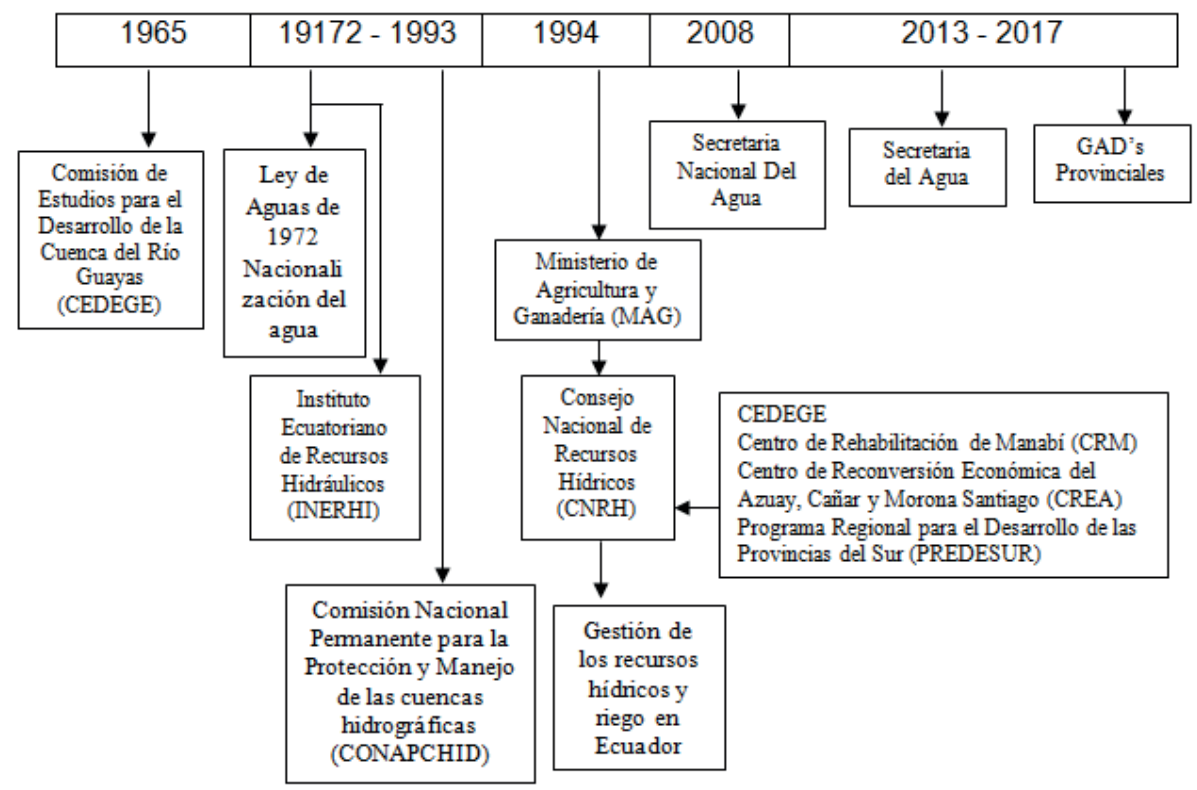

Fuente: Elaboración propia, 2021.

\section{Figura VIII: Institucionalidad en el manejo de la cuenca del río Guayas}

La cuenca del río Guayas, contempla proyectos de riego que han sido construido desde la década de los '80 hasta el año 2015, con fondos públicos. En la Tabla 2, se pueden observar sus características principales y en la Figura IX su ubicación espacial dentro de la cuenca del río Guayas. 
Gestión integrada de recursos hídricos y gobernanza: Subcuenca del río Vinces, provincia Los Ríos-Ecuador

Muñoz Marcillo, José Luis y Bustos Cara, Roberto

\section{Tabla 2}

Proyectos de riego y drenaje existentes en cuenca del rio guayas

\begin{tabular}{|c|c|c|c|c|c|c|}
\hline $\begin{array}{l}\text { NOMBRE } \\
\text { DEL PRO- } \\
\text { YECTO }\end{array}$ & UBICACIÓN & $\begin{array}{l}\text { AÑO DE } \\
\text { INAGU } \\
\text { RACIÓN }\end{array}$ & $\begin{array}{l}\text { SUPERFICIE } \\
\text { PROYECTA- } \\
\text { DA DE RIE- } \\
\text { GO (HA) }\end{array}$ & $\begin{array}{l}\text { SUPERFICIE } \\
\text { REAL DE RIE- } \\
\text { GO }\end{array}$ & \multicolumn{2}{|c|}{$\begin{array}{l}\text { ESTADO Y FUNCIONA- } \\
\text { LIDAD }\end{array}$} \\
\hline \multirow{5}{*}{ DAUVIN } & \multirow{5}{*}{$\begin{array}{l}\text { PROVIN- } \\
\text { CIAS DE } \\
\text { GUAYAS Y } \\
\text { LOS RÍOS }\end{array}$} & \multirow{5}{*}{2015} & \multirow{5}{*}{166000} & \multirow{5}{*}{60000} & Estado: & Bueno \\
\hline & & & & & Operando: & $\mathrm{Si}$ \\
\hline & & & & & $\begin{array}{l}\text { Área de riego } \\
\text { demarcada: }\end{array}$ & $\mathrm{Si}$ \\
\hline & & & & & $\begin{array}{l}\text { Canal de riego } \\
\text { construido: }\end{array}$ & $\mathrm{Si}$ \\
\hline & & & & & $\begin{array}{l}\text { Mantenimiento } \\
\text { Estatal: }\end{array}$ & $\mathrm{Si}$ \\
\hline \multirow{5}{*}{ CATARAMA } & \multirow{5}{*}{$\begin{array}{l}\text { VENTANAS } \\
\text { URDANETA } \\
\text { PUEBLO- } \\
\text { VIEJO }\end{array}$} & \multirow{5}{*}{2001} & \multirow{5}{*}{5760} & \multirow{5}{*}{1700} & Estado: & Regular \\
\hline & & & & & Operando: & $\mathrm{Si}$ \\
\hline & & & & & $\begin{array}{l}\text { Área de riego } \\
\text { demarcada: }\end{array}$ & $\mathrm{Si}$ \\
\hline & & & & & $\begin{array}{l}\text { Canal de riego } \\
\text { construido: }\end{array}$ & $\mathrm{Si}$ \\
\hline & & & & & $\begin{array}{l}\text { Mantenimiento } \\
\text { Estatal: }\end{array}$ & $\mathrm{Si}$ \\
\hline \multirow{5}{*}{$\begin{array}{l}\text { QUINSALO } \\
\text { MA - LOMA } \\
\text { DE COCO }\end{array}$} & \multirow{5}{*}{$\begin{array}{l}\text { QUINSALO- } \\
\text { MA }\end{array}$} & \multirow{5}{*}{1988} & \multirow{5}{*}{1200} & \multirow{5}{*}{1000} & Estado: & Bueno \\
\hline & & & & & Operando: & $\mathrm{Si}$ \\
\hline & & & & & $\begin{array}{l}\text { Área de riego } \\
\text { demarcada: }\end{array}$ & No \\
\hline & & & & & $\begin{array}{l}\text { Canal de riego } \\
\text { construido: }\end{array}$ & $\mathrm{Si}$ \\
\hline & & & & & $\begin{array}{l}\text { Mantenimiento } \\
\text { Estatal: }\end{array}$ & No \\
\hline \multirow{5}{*}{ ВАВАНОYО } & \multirow{5}{*}{ BABAHOYO } & \multirow{5}{*}{1982} & \multirow{5}{*}{9000} & \multirow{5}{*}{5000} & Estado: & Bueno \\
\hline & & & & & Operando: & $\mathrm{Si}$ \\
\hline & & & & & $\begin{array}{l}\text { Área de riego } \\
\text { demarcada: }\end{array}$ & $\mathrm{Si}$ \\
\hline & & & & & $\begin{array}{l}\text { Canal de riego } \\
\text { construido: }\end{array}$ & $\mathrm{Si}$ \\
\hline & & & & & $\begin{array}{l}\text { Mantenimiento } \\
\text { Estatal: }\end{array}$ & No \\
\hline \multirow{5}{*}{$\begin{array}{l}\text { DAULE } \\
\text { PERIPA }\end{array}$} & \multirow{5}{*}{$\begin{array}{l}\text { SECTOR NOR } \\
\text { OCCIDENTAL } \\
\text { DE PRO- } \\
\text { VINCIA DEL } \\
\text { GUAYAS Y } \\
\text { MANABI }\end{array}$} & \multirow{5}{*}{1982} & \multirow{5}{*}{100000} & \multirow{5}{*}{17000} & Estado: & Regular \\
\hline & & & & & Operando: & $\mathrm{Si}$ \\
\hline & & & & & $\begin{array}{l}\text { Área de riego } \\
\text { demarcada: }\end{array}$ & No \\
\hline & & & & & $\begin{array}{l}\text { Canal de riego } \\
\text { construido: }\end{array}$ & No \\
\hline & & & & & $\begin{array}{l}\text { Mantenimiento } \\
\text { Estatal: }\end{array}$ & No \\
\hline
\end{tabular}




\section{Cont... Tabla 2}

\begin{tabular}{|c|c|c|c|c|c|c|}
\hline \multirow{5}{*}{ PACALORI } & \multirow{5}{*}{$\begin{array}{c}\text { SECTOR SUR } \\
\text { OCCIDENTAL, } \\
\text { CENTRAL Y } \\
\text { SUR DE LA } \\
\text { PROVINCIA } \\
\text { DE LOS RÍOS }\end{array}$} & \multirow{5}{*}{$\begin{array}{c}\text { EXISTE } \\
\text { SOLO } \\
\text { ESTUDIO }\end{array}$} & \multirow{5}{*}{178000} & \multirow{5}{*}{$\begin{array}{c}\text { EXISTE SOLO } \\
\text { ESTUDIO }\end{array}$} & Estado: & N.A. \\
\hline & & & & & Operando: & N.A. \\
\hline & & & & & $\begin{array}{l}\text { Área de riego } \\
\text { demarcada: }\end{array}$ & N.A. \\
\hline & & & & & $\begin{array}{l}\text { Canal de riego } \\
\text { construido: }\end{array}$ & N.A. \\
\hline & & & & & $\begin{array}{l}\text { Mantenimiento } \\
\text { Estatal: }\end{array}$ & N.A. \\
\hline
\end{tabular}

Fuente: Elaboración propia, 2021.

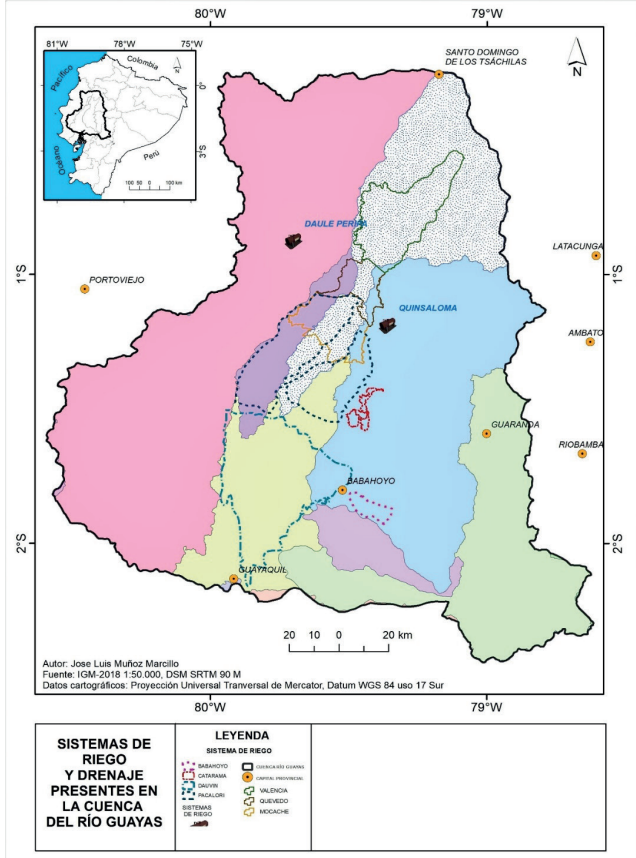

Fuente: Elaboración propia, 2021.

Figura IX: Ubicación de proyectos de riego y drenaje de la cuenca del Guayas 


\subsection{Cuenca del río Vinces}

Del total del agua de riego utilizada en la cuenca del río Vinces correspondiente a $538,35 \mathrm{hm} 3$ (ver Figura X), el $40 \%$ la consume el monocultivo de banano, seguido por un $20 \%$ del de cacao y un $5 \%$ por el monocultivo de palma aceitera, estos tres monocultivos mencionados destacan de los cultivos invernales de arroz y maíz, dado que a diferencia de estos dos cultivos de ciclo corto, son cultivos cuya mayor productividad se concentra en los ocho meses de verano y ocupan importantes tasas de consumo de agua, así por ejemplo de acuerdo a lo manifestado por productores agrícolas, una ha de banano consume en promedio $12.000, \mathrm{~m} 3$ de agua por año, en el caso del cacao, su consumo de agua para riego en relación al banano es de un $40 \%$, mientras que la palma aceitera, corresponde a un $35 \%$.

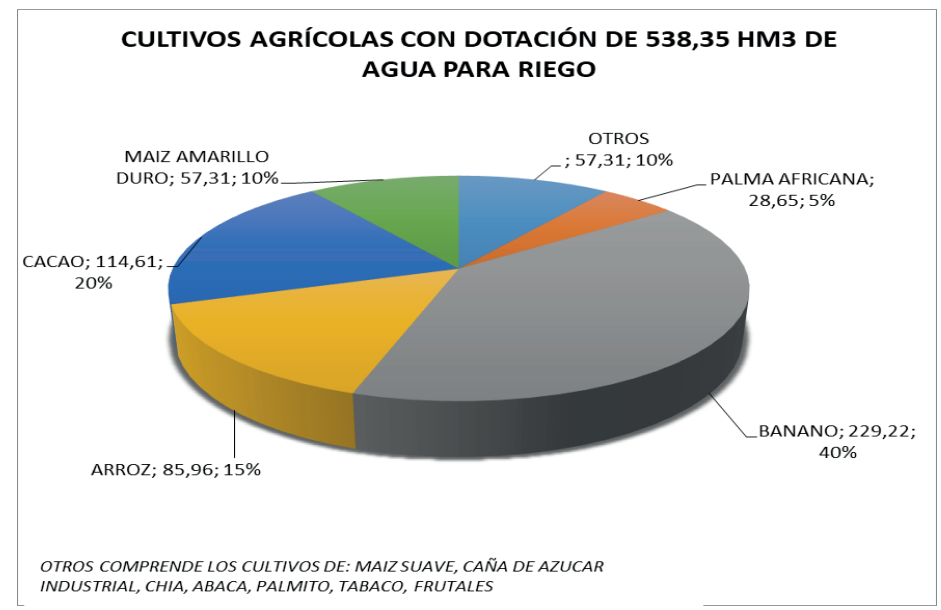

Fuente: Elaboración propia, 2021.

\section{Gráfico I: Cultivos agrícolas con dotación de $538,35 \mathrm{hm} 3$ de agua para riego}

La concesión de caudales para riego cuando es menor a los 5 litros/segundo en la cuenca del río Vinces, la otorga la Oficina Técnica de la demarcación Hidrográfica del Guayas, ubicada en la ciudad de Quevedo y que es administrada por la Secretaría del Agua (SENAGUA). En el Gráfico II, se pueden apreciar que de los años registrados las concesiones no son constantes, lo cual se debe a que no existe un registro real de todas las concesiones, dado que muchas se realizan sin solicitar la autorización respectiva, y si a esto se le suma, que en la actualidad no existen mecanismos de control de consumo de caudal como medidores, la situación se presenta más complicada con el fin de realizar un verdadero control de los caudales concedidos 


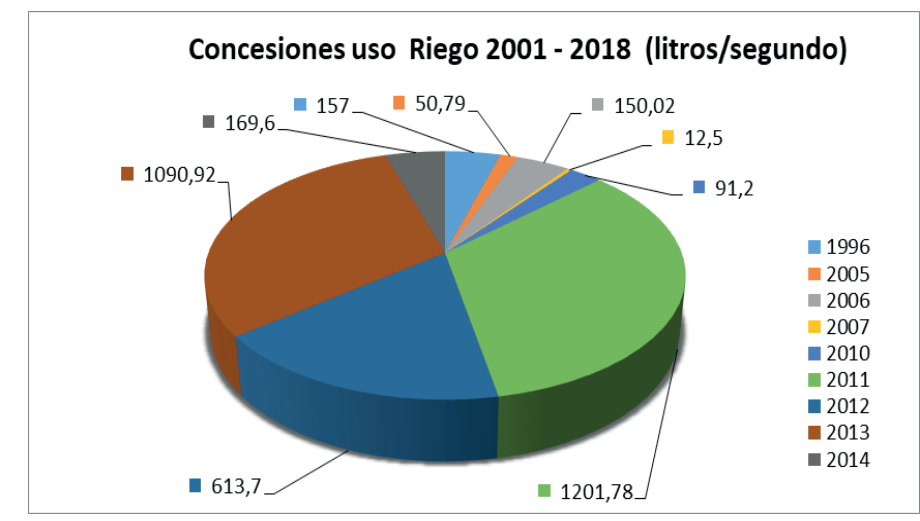

Fuente: Elaboración propia, 2021.

\section{Gráfico II: Concesiones de uso de riego 2001-2018 (litros/segundo)}

La oficina matriz de atención al cliente de SENAGUA, ubicada en la ciudad de Guayaquil correspondiente a la demarcación hidrográfica del Guayas, le corresponde realizar las concesiones de caudales mayores a 5 litros/segundo que en su gran parte se relacionan con productores bananeros, cacaoteros y palmicultores. Se puede notar en el Gráfico III, que del año 2011 al año 2014 se registran los mayores volúmenes de caudales autorizados, debido a la recién creada Secretaría Nacional del Agua (SENAGUA), institución descentralizada que imprimió control importante en la distribución de agua para riego en el Ecuador.

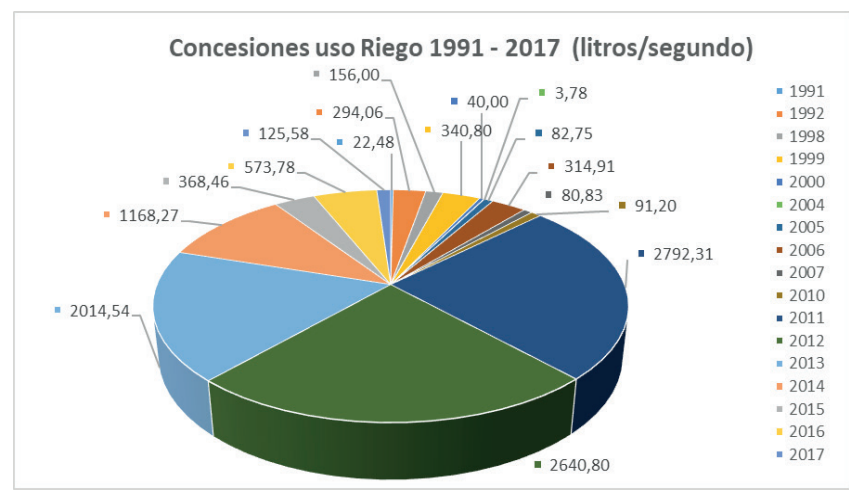

Fuente: Elaboración propia, 2021.

Gráfico III: Concesiones de uso de riego 1991-2017 (litros/segundo) 
Gestión integrada de recursos hídricos y gobernanza: Subcuenca del río Vinces, provincia Los Ríos-Ecuador

Muñoz Marcillo, José Luis y Bustos Cara, Roberto

De acuerdo con Muñoz, Gentili y Bustos (2020): El rápido crecimiento demográfico y la creciente demanda internacional de productos tropicales han propiciado la conversión de extensas áreas de tierra para la producción agrícola intensiva de varios monocultivos en el
Ecuador" (p.92). En ese sentido, la estructura de la tenencia de la tierra rural en los cantones Valencia, Quevedo y Mocache de acuerdo a la superficie total por hectárea se presenta en la Tabla 3.

Tabla 3

Estructura de tenencia de la tierra rural por superficie de lotes

\begin{tabular}{ccccccccccc}
\hline $\begin{array}{c}\text { Área Ha } \\
\text { Cantón }\end{array}$ & $\mathbf{0 - 1 0} \mathbf{~ H a}$ & $\begin{array}{c}\mathbf{1 0 - 2 0} \\
\mathbf{H a}\end{array}$ & $\mathbf{2 0 - 5 0 ~ H a}$ & $\begin{array}{c}\mathbf{5 0 - 1 0 0} \\
\mathbf{H a}\end{array}$ & $\begin{array}{c}\mathbf{1 0 0 - 2 0 0} \\
\mathbf{H a}\end{array}$ & $\begin{array}{c}\text { Más de } \mathbf{2 0 0} \\
\mathbf{H a}\end{array}$ & Total Ha & $\begin{array}{c}\text { Área Real } \\
\text { Rural Ha }\end{array}$ & $\begin{array}{c}\text { \% } \\
\text { Muestreo }\end{array}$ \\
\hline VALENCIA & $2.171,08$ & $2.883,57$ & $5.290,10$ & $5.943,58$ & $7.599,47$ & $32.990,26$ & $56.878,06$ & $9.7150,00$ & 58,55 \\
QUEVEDO & $1.734,48$ & $1.578,00$ & $2.405,99$ & $4.213,76$ & $2.703,00$ & $19.000,77$ & $31.636,00$ & $32.105,00$ & 98,54 \\
MOCACHE & $2.552,79$ & $2.127,39$ & $2.995,00$ & $3.045,79$ & $2.956,04$ & $8.730,46$ & $22.407,47$ & $56.490,00$ & 39,66 \\
\hline
\end{tabular}

Fuente: Elaboración propia, 2021.

Asimismo, la estructura de la tenencia de la tierra rural en el cantón Valencia, Quevedo y Mocache, expresada en porcentajes en relación a las diferentes categorías de superficie correspondiente a la parte alta de la cuenca del rio Vinces, se presenta en el Gráfico IV. La Organización de las Naciones Unidas para la Alimentación y la Agricultura (FAO, 2008) sostiene que: "La tenencia de la tierra y los derechos de propiedad sobre ella resultan indispensables no sólo para el desarrollo económico y social sostenible y la mitigación de la pobreza, sino también para la gestión del riesgo" (p.4), así como de la disminución de los efectos en relación a los desastres naturales.

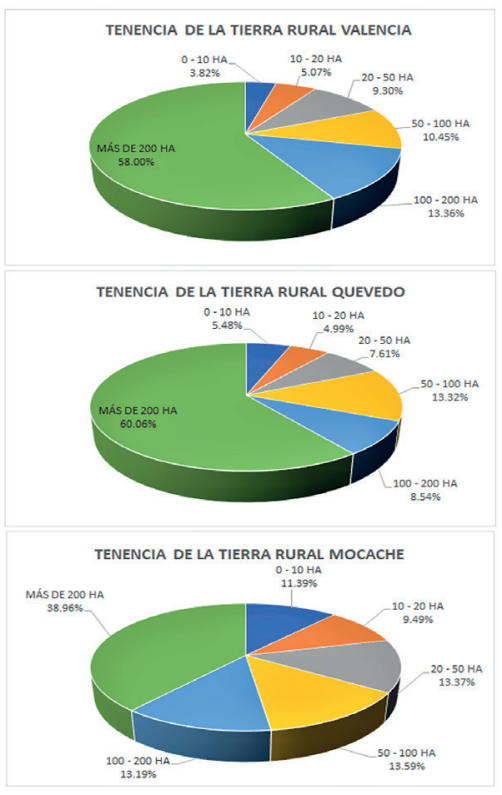

Fuente: Elaboración propia, 2021.

\section{Gráfico IV: Tenencia de la tierra rural en el cantón Valencia, Quevedo y Mocache}


En el Gráfico V, se muestra la estructura de la tenencia de la tierra rural en los cantones
Valencia, Quevedo y Mocache, de acuerdo a las interrelaciones por categorías de superficie.
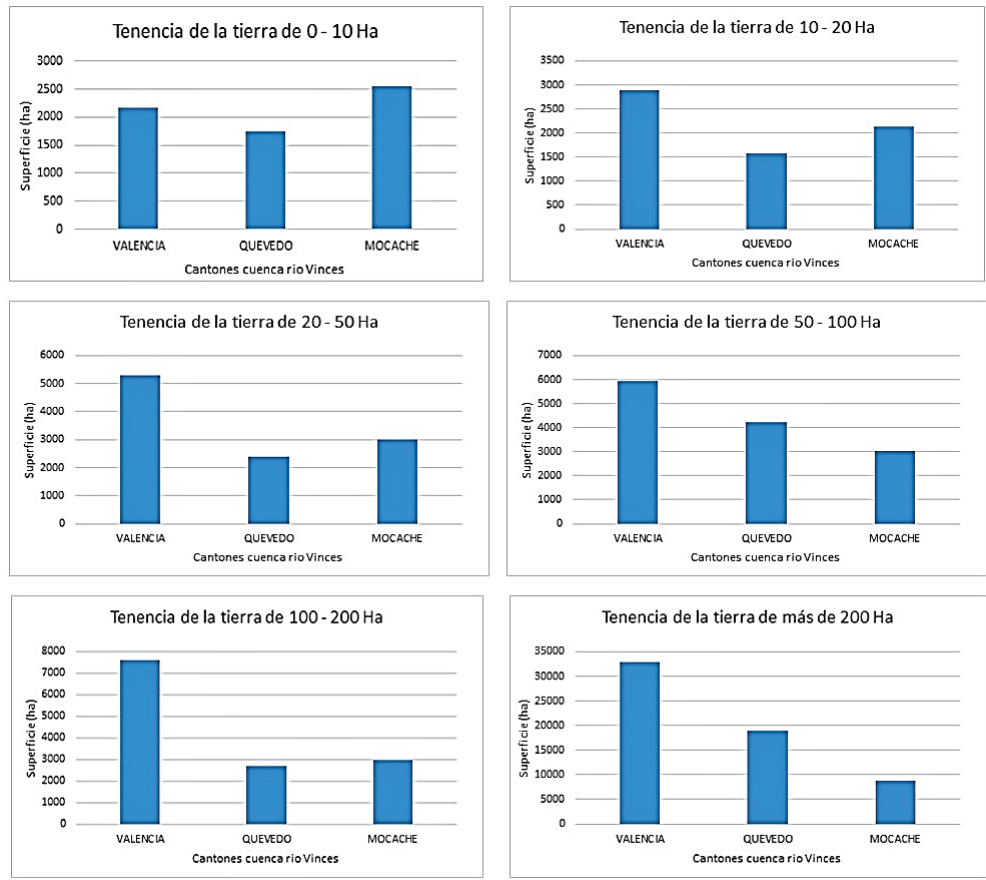

Fuente: Elaboración propia, 2021.

\section{Gráfico $V$ : Tenencia de la tierra por superficie de lotes en zonas de la cuenca del río Vinces}

La tenencia de la tierra en la parte alta, media y baja de la cuenca del río Vinces de acuerdo a la superficie total que abarcan los predios por su tamaño en hectáreas, muestra que en el cantón Mocache predominan las superficies ocupadas por lotes de entre 20 a 100 ha. En la parte alta de la cuenca del río Vinces, representada por el cantón Valencia existe un predominio de lotes de entre 50 a 200 ha, debido a que en este cantón se concentran importantes compañías y productores independientes de banano de exportación que realizan una actividad agrícola intensiva a gran escala. En la parte media de la cuenca predomina la superficie cubierta por lotes de entre 50 a 100, ha dado que este cantón mayormente urbano es menor a un 50\% y $25 \%$ de la superficie de los cantones Mocache y Valencia, respectivamente,

La estructura de la tenencia de la tierra rural en los cantones Valencia, Quevedo y Mocache, de acuerdo al número de lotes dentro de las diferentes categorías de superficie por ha, se presentan en la Tabla 4. 
Gestión integrada de recursos hídricos y gobernanza: Subcuenca del río Vinces, provincia Los Ríos-Ecuador

Muñoz Marcillo, José Luis y Bustos Cara, Roberto

\section{Tabla 4}

\section{Estructura de la tenencia de la tierra rural por números de lotes}

\begin{tabular}{|c|c|c|c|c|c|c|c|c|c|}
\hline $\begin{array}{l}\text { Arrea } \mathrm{Ha} \\
\text { Cantón }\end{array}$ & $\begin{array}{c}\text { Lotes } \\
0-10 \\
\mathrm{Ha}\end{array}$ & $\begin{array}{c}\text { Lotes } \\
10-20 \\
\mathrm{Ha}\end{array}$ & $\begin{array}{c}\text { Lotes } \\
20-50 \\
\mathrm{Ha} \\
\end{array}$ & $\begin{array}{c}\text { Lotes } \\
50-100 \\
\mathrm{Ha}\end{array}$ & $\begin{array}{c}\text { Lotes } \\
100-200 \\
\mathrm{Ha}\end{array}$ & $\begin{array}{c}\text { Lotes } \\
\mathbf{2 0 0}-\mathbf{5 0 0} \\
\mathbf{H a}\end{array}$ & $\begin{array}{c}\text { Lotes } \\
500- \\
2000 \mathrm{Ha}\end{array}$ & $\begin{array}{l}\text { Total } \\
\text { Lotes }\end{array}$ & $\begin{array}{c}\text { Area Total } \\
\mathrm{Ha}\end{array}$ \\
\hline VALENCIA & 6.470 & 907 & 765 & 209 & 90 & 24 & 11 & 8.476 & $97.150,00$ \\
\hline QUEVEDO & 5.608 & 312 & 183 & 50 & 13 & 10 & 0 & 6.176 & $32.105,00$ \\
\hline MOCACHE & 11.446 & 755 & 416 & 91 & 14 & 8 & 4 & 12.734 & $56.490,00$ \\
\hline
\end{tabular}

Fuente: Elaboración propia, 2021.

De igual manera, la estructura de la tenencia de la tierra rural en el cantón Valencia, expresada en porcentajes en relación al número de lotes dentro de las diferentes categorías de superficie correspondiente a la parte alta de la cuenca del rio Vinces, se muestra en el Gráfico VI.

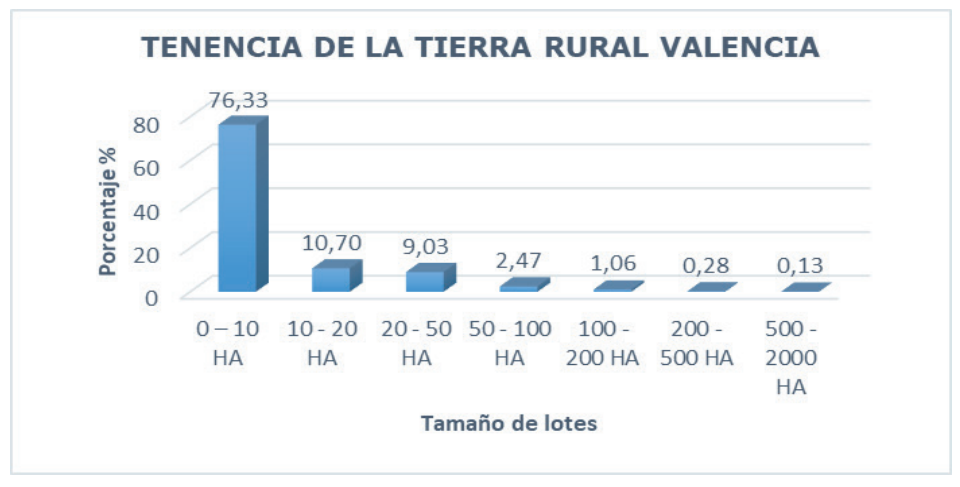

Fuente: Elaboración propia, 2021.

\section{Gráfico VI: Tenencia de la tierra rural en el cantón Valencia}

La estructura de la tenencia de la tierra rural en el cantón Quevedo, expresada en porcentajes en relación al número de lotes dentro de las diferentes categorías de superficie correspondiente a la parte media de la cuenca del rio Vinces, se presenta en el Gráfico VII. 


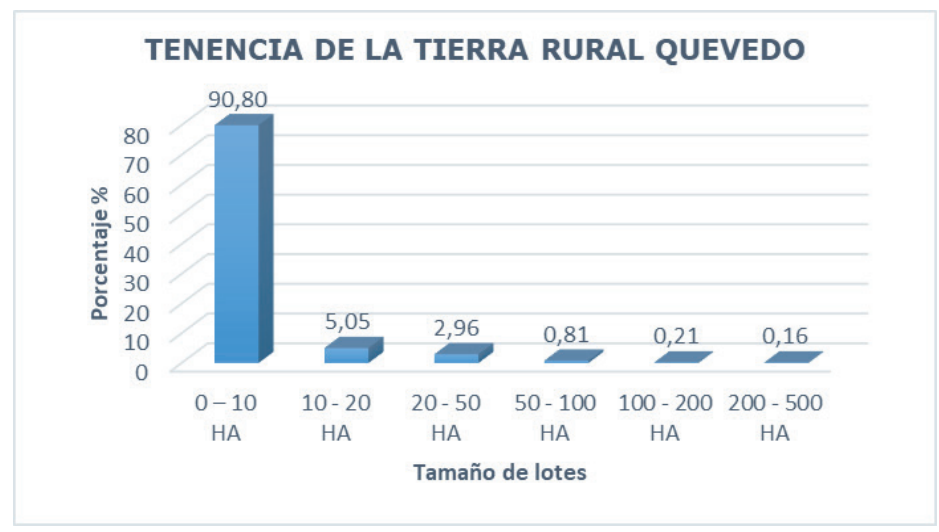

Fuente: Elaboración propia, 2021.

\section{Gráfico VII: Tenencia de la tierra rural en el cantón Quevedo}

Asimismo, la estructura de la tenencia de la tierra rural en el cantón Mocache, expresada en porcentajes en relación al número de lotes dentro de las diferentes categorías de superficie correspondiente a la parte baja de la cuenca del rio Vinces, se aprecia en el Gráfico VIII.

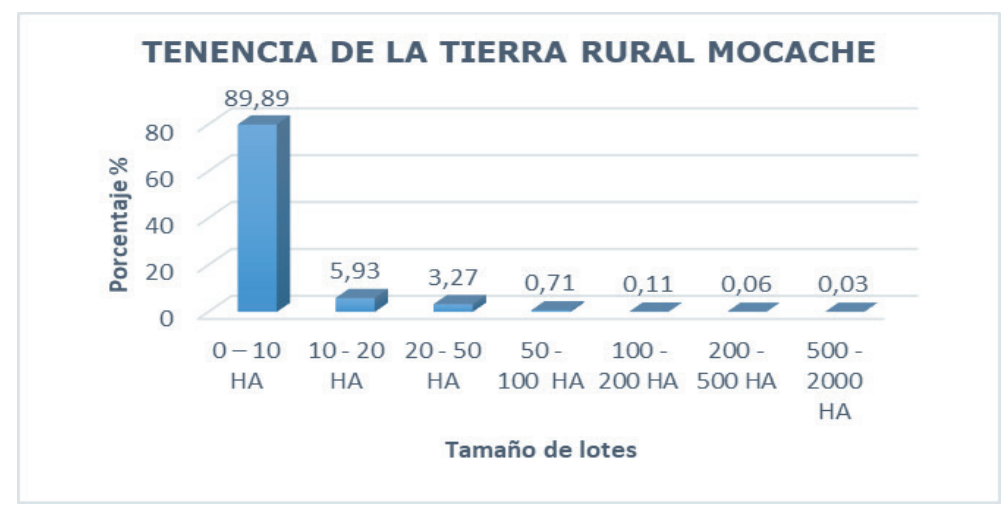

Fuente: Elaboración propia, 2021.

Gráfico VIII: Tenencia de la tierra rural en el cantón Mocache 
Gestión integrada de recursos hídricos y gobernanza: Subcuenca del río Vinces, provincia Los Ríos-Ecuador

Muñoz Marcillo, José Luis y Bustos Cara, Roberto

En este sentido, en el Gráfico IX se presenta la estructura de la tenencia de la tierra rural en los cantones Valencia, Quevedo y
Mocache, de acuerdo a las interrelaciones por número de lotes dentro de las categorías de superficie.
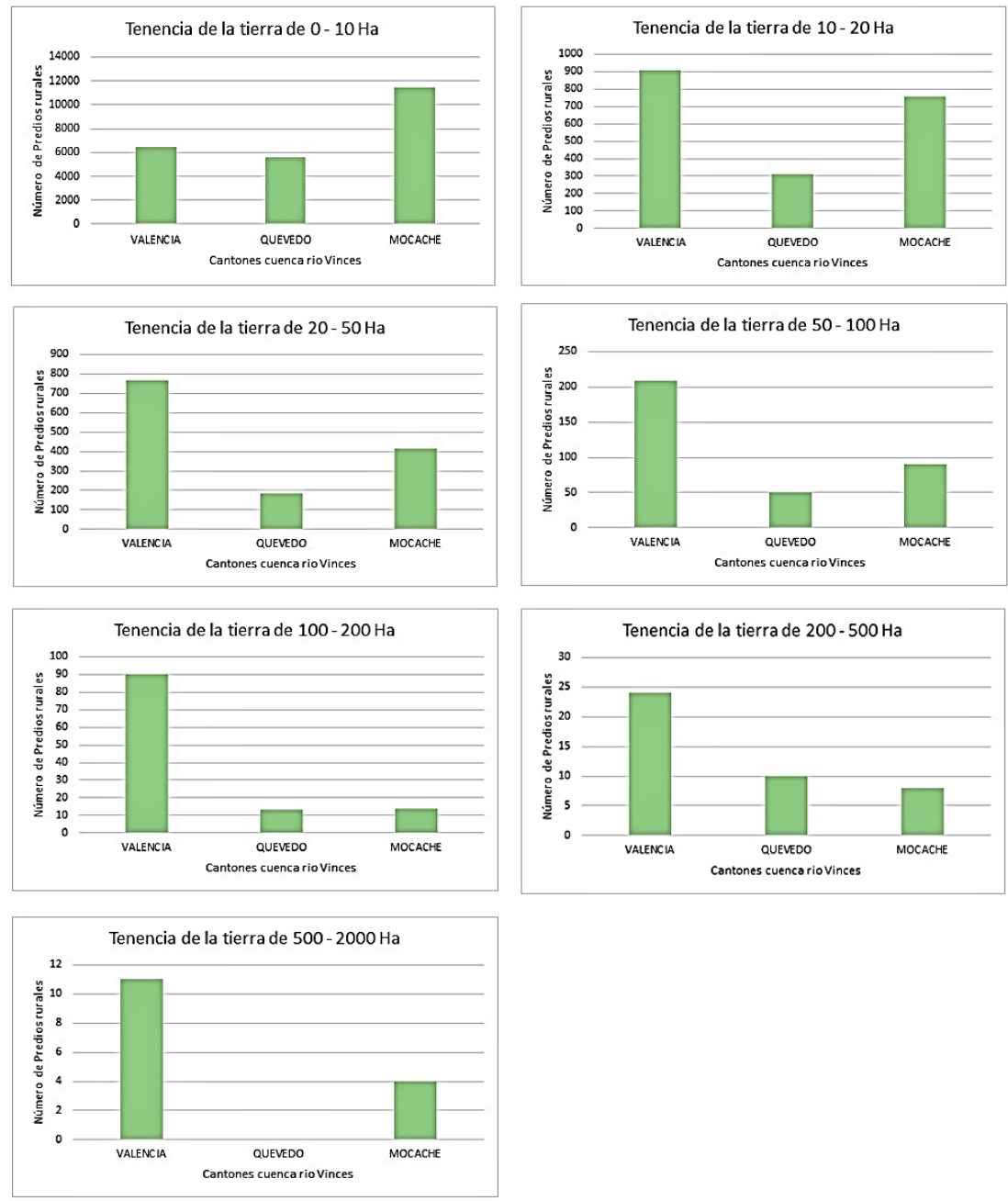

Fuente: Elaboración propia, 2021.

\section{Gráfico IX: Tenencia de la tierra por número de lotes en zonas de la cuenca del} rio Vinces 
La gestión del recurso hídrico en la subcuenca del río Vinces, sufre una fragmentación debido al variado número de entidades que tienen ciertos niveles de competencias para el manejo de los proyectos de riego, siendo el caso del MAGAP, el MAE, GAD's provinciales y GAD's cantonales, derivando esta situación en un manejo deficiente del recurso hídrico que se puede comprobar en la realidad del campo donde son recurrentes los conflictos por el uso de agua para riego, sobre todo con los pequeños productores. Por lo cual, tal como lo señala Gaybor (2008), Ecuador, demanda cambios importantes en el acceso al agua, en el manejo sostenible de los recursos, aprovechamiento eficiente de los mismos, así como, en el sistema institucional y normativo del país.

\section{Conclusiones}

A escala del río Guayas, se observó una importante gradiente altitudinal que marca su variada topografía y edafología, permitiendo un uso diverso del suelo que ha derivado en la distribución de la producción agrícola en muchos cultivos, entre los que destacan los monocultivos de exportación como el cacao, banano, palma aceitera, arroz y maíz, que constituyen la fuente de importantes ingresos de divisas para el país. Seguido de la producción agrícola, se pudo apreciar una importante actividad pecuaria principalmente en la zona norte de la cuenca, dedicada al ganado de leche y en menor medida de carne; es importante destacar, que la presencia de bosques nativos en la cuenca es menor, habiendo en las dos últimas décadas aumentado significativamente las plantaciones de especies forestales exóticas como la teca, gmelina y balsa.

En la última década, la administración de la cuenca del río Guayas ha sufrido un cambio administrativo importante. No obstante, de la visión holística que se le ha dado a la gestión de los recursos hídricos, mediante la delimitación de las demarcaciones hidrográficas, la cuenca del río Guayas sigue presentando conflictos en el uso del agua de riego en los usuarios finales, en virtud que sigue el acaparamiento del agua por los grupos de poder en desmedro de los pequeños agricultores, que pese a sus reclamos no reciben soluciones efectivas y los mismos solo se quedan en el aún latente aparato burocrático.

La cuenca del río Vinces, actualmente como parte de la Demarcación Hidrográfica del Guayas, ha ganado cierta autonomía administrativa que no tenía cuando dependía de la Comisión de Estudios para el Desarrollo de la Cuenca del río Guayas (CEDEGÉ). Sin embargo, el centralismo funcional del gobierno no ha permitido fortalecer una verdadera autonomía sobre todo a nivel económico, que potencie una gobernanza de su territorio.

El Gobierno Provincial de Los Ríos (GPDLR), tiene a cargo por mandato de ley los proyectos de riego y drenaje que se ejecuten en el territorio provincial que contiene toda el área agrícola productiva de la cuenca del río Vinces, por falta de recursos económicos el desempeño del GPDLR para proyectos de riego y drenaje en la cuenca del río Vinces, ha sido muy limitado, y considerando la falta de disponibilidad de agua para riego en la zona sur de la cuenca en los ocho meses de verano, el gobierno central tiene ya realizado los estudios del polémico proyecto insigne PACALORI que afectaría el centro - sur de la cuenca, prometiendo más conflictos que soluciones claras y que no se ha construido por falta de presupuesto estatal, dado que su construcción bordearía los cinco mil millones de dólares norte americanos.

El uso eficiente y equitativo del agua, asegurando la conservación y protección del recurso hídrico, se logra mediante una positiva gestión de la misma dado su efecto integrador tanto en el bienestar económico y social. Lamentablemente, el valor integral del agua no ha sido reconocido en la cuenca del río Guayas, donde ha prevalecido el factor económico sobre el bienestar social de toda la población, lo cual se puede comprobar, cuando se aprecia que los grandes grupos de poder económico utilizan elevados volúmenes de agua para el riego de sus monocultivos en 
Gestión integrada de recursos hídricos y gobernanza: Subcuenca del río Vinces, provincia Los Ríos-Ecuador

Muñoz Marcillo, José Luis y Bustos Cara, Roberto

desmedro de los pequeños agricultores y de la conservación del recurso hídrico, sin que exista una verdadera regulación estatal, así como pagando irrisorias tasas anuales por concepto de caudal que generalmente es sobre utilizado.

Finalmente, en Ecuador resulta prioritario repensar y redefinir con cuidado los roles de los diferentes niveles de gobierno, proponer nuevos modelos de gestión descentralizada de competencias, que establezcan las relaciones y articulaciones verticales, así como horizontales entre los actores tanto públicos como privados, al igual que nuevas modalidades de financiamiento. Por ello, el desarrollar modelos descentralizados de gobernanza y de gestión, que permitan la formulación de objetivos comunes y su implementación en los territorios, con herramientas e instrumentos para la puesta en escena de políticas públicas, es una apuesta técnica y viable de reforma del Estado.

\section{Referencias bibliográficas}

Aguirre, M. (2011). La cuenca hidrográfica en la gestión integrada de los recursos hídricos. Revista Virtual REDESMA, 5(1), 9-20. http://www.siagua.org/ sites/default/files/documentos/ documentos/cuencas $m$ aguirre.pdf

Asociación Mundial para el Agua (Global Water Partnership) - GWP (2000). Manejo Integrado de los Recursos Hidricos. TAC Background Papers No. 4. GWP. https://www.gwp. org/globalassets/global/toolbox/ publications/background-papers/04integrated-water-resourcesmanagement-2000-spanish.pdf

Barthe, L. (2001). La gouvernance locale des stratégies de développement territorial en milieu rural. Éléments de reflexionsur l'action organisationnelle territorialisée. Revista Universitaria de Geografía, 10(1 y 2), 73-85.
Budds, J. (2020). Gobernanza del agua y desarrollo bajo el mercado: Las relaciones sociales de control del agua en el marco del Código de Aguas de Chile. Investigaciones Geográficas, (59), 16-27. https://doi. org/10.5354/0719-5370.2020.57717

Bustos, R. (2014). Territorio y desarrollo rural. En Albaladejo Ch, Bustos Cara R y Gisclard M (comp). En C. Albaladejo, R. Bustos y M. Gisclard (Eds.), Transformaciones de la actividad agropecuaria de los territorios y de las políticas públicas: entrelazamientos de lógicas (pp. 263-274). Editorial de la Universidad del Sur.

Changjiang Insitute of Survey Planning Design and Research - CISPDR (2016a). Plan Nacional de Gestión Integrada e Integral de los Recursos Hídricos $y$ de las Cuencas y Microcuencas hidrográficas de Ecuador. CISPDR.

Changjiang Institute of Survey Planning Design and Research - CISPDR (2016b). Plan Hidráulico Regional de la Demarcación Hidrográfica Guayas. Memoria (version definitiva). CISPDR. http://suia.ambiente.gob. ec/files/MEMORIA \%20DH\%20 GUAYAS.pdf

Consorcio Camaren (22 de junio de 2012). Conclusiones del VII Encuentro Nacional del Foro de los Recursos Hídricos. Consorcio Camaren. https:// camaren.org/conclusiones-del-viiencuentro-nacional-del-foro-de-losrecursos-hidricos/

Contreras, P., y Montecinos, E. (2019). Democracia y participación ciudadana: Tipología y mecanismos para la implementación. Revista de Ciencias Sociales (Ve), XXV(2), 178-191.

Dourojeanni, A., Jouravlev, A., y Chávez, G. (2002). Gestión del agua a nivel de cuencas: Teoría y práctica. Naciones Unidas. $\quad$ https://repositorio.cepal. 
org/bitstream/handle/11362/6407/1/ $\underline{\text { S028593 es.pdf }}$

García, M. L., Carvajal, Y., y Jiménez, H. (2007). La gestión integrada de los recursos hídricos como estrategia de adaptación al cambio climático. Ingeniería y Competitividad, 9(1), 19-29. https://doi.org/10.25100/iyc. v9i1.2492

Gaybor, A. (2008). El despojo del agua y la necesidad de una transformación urgente. Consorcio CAMAREN.

Gómez, D., y Gómez, A. (2013). Ordenación territorial. Editorial Mundi-Prensa.

Instituto Geográfico Militar - IGM (2018). Obtención de cartografia básica oficial actualizada del pais multiescala. IGM. http://www.igm. gob.ec/work/files/lotaip/2018/julio/ proyecto multiescala.pdf

Instituto Oceanográfico de la Armada INOCAR (2010). Memoria Técnica de la comisión realizada en el área del Río Guayas sur. 29 de noviembre al 08 de diciembre del 2009. Instituto Oceanográfico de la Armada.

Integrated Watershed Management - IWM (2015). Protecting water resources and addressing environmental challenges. https://conservationontario.ca/policypriorities/integrated-watershedmanagement

Kooiman, J. (2005). Gobernar en gobernanza. En A. C. Martínez (Comp), En La gobernanza hoy: 10 textos de referencia (pp. 57-82). Editorial INAP.

Küffner, U. (2004). El proceso de la formulación de la política y estrategia de manejo de los recursos hídricos en el Ecuador. Asociación Mundial del Agua (GWP). Comisión Económica para América Latina y el Caribe (CEPAL).

Kumar, B., Kumar, P., Saraswat, C.,
Chakraborty, S., y Gautam, A. (2021). Water security in a changing environment: Concept, challenges and solutions. Water, 13(4), 490. https:// doi.org/10.3390/w13040490Lemos, M. C., y Agrawal, A. (2006). Environmental governance. Annual Review of Environment and Resources, 31, 297-325. https://doi.org/10.1146/ annurev.energy.31.042605.135621

Martínez, Y., y Villalejo, V. M. (2018). La gestión integrada de los recursos hídricos: una necesidad de estos tiempos. Ingeniería Hidráulica $y$ Ambiental, 39(1), 58-72.

Mersha, A. N., De Fraiture, C., Masih, I., y Alamirew, T. (2021). Dilemmas of integrated water resources management implementation in the Awash River Basin, Ethiopia: irrigation development versus environmental flows. Water and Environment Journal, 35(1), 402-416. https://doi.org/10.1111/wej.12638

Ministerio de Agricultura y Ganadería MAGAP (2015). Land use and land cover map. Years 2014-2015, 1:25,000 Scale. $\quad$ http://ide.sigtierras.gob.ec/ geoportal/

Muñoz, J. L., Gentili, J., y Bustos, R. (2020). Uso agrícola del suelo y demanda de agua para riego en la cuenca del río Vinces (Ecuador) durante el período 1990-2014. Investigaciones Geográficas, (59), 91-104. https://doi. org/10.5354/0719-5370.2020.56958

Natera, A. (2004). La noción de gobernanza como gestión pública participativa $y$ reticular. Universidad Carlos III de Madrid, Departamento de Ciencias Política y Sociología.

Ngene, B. U., Nwafor, C. O., Bamigboye, G. O., Ogbiye, A. S., Ogundare, J. O., y Akpan, V. E. (2021). Assessment of water resources development and exploitation in Nigeria: A 
Gestión integrada de recursos hídricos y gobernanza: Subcuenca del río Vinces, provincia Los Ríos-Ecuador

Muñoz Marcillo, José Luis y Bustos Cara, Roberto

review of integrated water resources management approach, Heliyon, 7(1), e05955. https://doi.org/10.1016/j. heliyon.2021.e05955

Oficina de Evaluación y Supervisión Ove (2012). Evaluación sectorial $y$ temática. El reto del Manejo Integrado de Cuencas Hidrográficas: Análisis de la acción del BID en programas de manejo de cuencas 1989-2010. Banco Interamericano de Desarrollo (BID). https://publications. iadb.org/publications/spanish/ document/Nota-tem $\%$ C3\%A1ticaEl-reto-del-manejo-integrado-decuencas-hidrogr\%C3\%A1ficas;A n \% C 3\% A 11 is is - de - $1 \mathrm{a}-$ acci $\%$ C $3 \%$ B $3 n-d$ e $1-$ Bancoen-programas-de-manejo-de-cuencas-1989-2010.pdf

Organización de las Naciones Unidas para la Alimentación y la Agricultura - FAO (2008). El Ecuador: Un país con elevada vulnerabilidad. En FAO (Ed.), En tierra segura: desastres naturales y tenencia de la tierra (pp. 26-38). FAO, Uni-Habitat. http://www.fao.org/3/ i1255b/i1255b.pdf

Organización de las Naciones Unidas para la Alimentación y la Agricultura - FAO (2009). ¿Por qué invertir en ordenación de las cuencas hidrográficas? FAO. http://www.fao. org/3/a1295s/a1295s00.pdf

Özerol, G., Bressers, H., y Coenen, F. (2012). Irrigated agriculture and environmental sustainability: an alignment perspective. Environmental Science \& Policy, 23, 57-67. https:// doi.org/10.1016/j.envsci.2012.07.015

Pahl-Wostl, C., Arthington, A., Bogardi, J., Bunn, S. E., Hoff, H., Lebel, L., Nikita, E., palmer, N., Poff, L. N., Richards, K., Schluter, M., Schulze, R., StHilaire, A., Tharme, R., Tsegai, D. (2013). Environmental flows and wáter governance: managing sustainable water uses. Current Opinion in Environmental Sustainability, 5(3-4), 341-351. https://doi.org/10.1016/j. cosust.2013.06.009

Pierre, J., y Peters, B. G. (2000). Governance, politics and the state. Palgrave.

Postel, S. L. (2000). Entering an era of water scarcity: The challenges ahead. Ecological Applications, 10(4), 941948. $\quad$ https://doi.org/10.1890/10510761(2000)010[0941:EAEOWS]2.0. $\underline{\mathrm{CO} ; 2}$

Ramos-Soto, A. L., Londoño, D. C., Sepulveda-Aguirre, J., y MartínezJiménez, R. (2020). Gestión integral e integrada: Experiencia de las empresas en México. Revista de Ciencias Sociales (Ve), XXVI(3), 31-44. https:// doi.org/10.31876/rcs.v26i3.33229

Red Internacional para el Desarrollo de Capacidades en la Gestión Integrada del Recurso Hídrico - Cap-Net (2008). Gestión Integrada de los Recursos Hidricos para organizaciones de cuencas fluviales. Manual de capacitación. Cap-Net. https:// gestionsostenibledelagua.files. wordpress.com/2010/10/2008-girhpara-organizaciones-de-cuencasfluviales.pdf

Red Internacional para el Desarrollo de Capacidades en la Gestión Integrada del Recurso Hídrico (Cap-Net) y la Asociación Mundial para el Agua (GWP) (2005). Planes de Gestión Integrada del Recurso Hídrico: Módulo de capacitación y guía operacional. Cap-Net https://www. gwp.org/globalassets/global/gwpcam files/manual-planes-girh.pdf

Rockström, J., Williams, J., Daily, G., Noble, A., Matthews, N., Gordon, L., Wetterstrand, H., DeClerck, F., Shah, M., Steduto, P., De Fraiture, 
C., Hatibu, N., Unver, O., Bird, J., Sibanda, L., y Smith, J. (2017). Sustainable intensification of agriculture for human prosperity and global sustainability. Ambio, 46(1), 4-17. https://doi.org/10.1007/s13280016-0793-6

Rosegrant, M. W., Ringler, C., y Zhu, T. (2009). Water for agriculture: maintaining food security under growing scarcity. Annual Review of Environment and Resources, 34, 205222. https://doi.org/10.1146/annurev. environ.030308.09051

Sánchez, M. L., y Castañón, J. C. (2019). Gobernanza en la educación superior de México: Caso Universidad Autónoma de Tamaulipas. Revista de Ciencias Sociales (Ve), XXV(3), 59-71.

Secretaría Nacional del Agua - SENAGUA (2017). Boletín de la Estadística Sectorial del Agua. SENAGUA. https://docplayer.es/88162270Megaproyectos-balances-boletin-dela-estadisticas-estadistica-sectorialdel-caudales-agua.html

Secretaría Nacional del Agua y Servicio Alemán de Cooperación Técnico Social SENAGUA-DED (2009). Estudio exploratorio: Problemática y conflictos sobre los recursos hídricos por efectos del cambio climático. SENAGUA-DED. https://docplayer. es/15524027-Estudio-exploratorioproblematica-y-conflictos-sobre-losrecursos-hidricos-por-efectos-del- cambio-climatico-resumen-ejecutivo. $\underline{\mathrm{html}}$

Secretaría Nacional de Planificación y Desarrollo - SENPLADES (2017). Estructura Orgánica de la Función Ejecutiva de la República del Ecuador. SENPLADES. http:// www.planificacion.gob.ec/wpcontent/uploads/downloads/2017/10/ Organigram a - FuncionEjecutiva_25-05-2017.pdf

Smith, M., y Clausen, T. J. (2018). Revitalising IWRM for the 2030 agenda. World Water Council.

Van-Rijswick, M., Edelenbos, J., Hellegers, P., Kok, M., y Kuks, S. (2014). Ten building blocks for sustainable water governance: An integrated method to assess the governance of water. Water International, 39, 725-742. https://doi. org $/ 10.1080 / 02508060.2014 .951828$

Wheater, H., y Evans, E. (2009). Land use, water management and future flood risk. Land Use Policy, 26(S-1), S251-S264. https://doi.org/10.1016/j. landusepol.2009.08.019

Wiek, A., y Larson, K. L. (2012). Water, people, and sustainability-A systems framework for analyzing and assessing water gover-nance regimes. Water Resources Management, 26, 31533171. https://doi.org/10.1007/s11269$\underline{012-0065-6}$ 